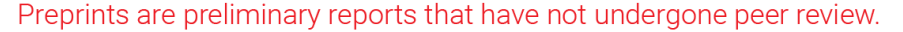 They should not be considered conclusive, used to inform clinical practice, or referenced by the media as validated information. \\ LSALO-BP: A Hybrid Model for Children Adulthood Height Prediction
}

\section{Keji Mao}

Zhejiang University of Technology

\section{Lijian Chen}

Zhejiang University of Technology

Xinben Fan ( $D$ 892902601@qq.com )

Zhejiang University of Technology

Jiafa Mao

Zhejiang University of Technology

\section{Xiaolong Zhou}

Quzhou University

\section{Kai Fang}

Macau University of Science and Technology https://orcid.org/0000-0002-6247-5250

\section{Research Article}

Keywords: adult height prediction of children, location strategy, improved Ant lion optimizer, bp neural network, prediction mode

Posted Date: January 6th, 2022

DOI: https://doi.org/10.21203/rs.3.rs-1212743/v1

License: (c) (i) This work is licensed under a Creative Commons Attribution 4.0 International License.

Read Full License 


\title{
LSALO-BP: A Hybrid Model for Children Adulthood Height Prediction
}

\author{
Keji Mao ${ }^{1}$, Lijian Chen ${ }^{1}$, Xinben Fan ${ }^{1}$, Jiafa $\mathrm{Mao}^{1}$, Xiaolong \\ Zhou $^{2}$ and Kai Fang ${ }^{2,3^{*}}$ \\ $1^{*}$ College of Computer Science and Technology, Zhejiang \\ University of Technology, Xihu, Hangzhou, 310023, Zhejiang, \\ China. \\ ${ }^{2}$ College of Electrical and Information Engineering, Quzhou \\ University, Kechen, Quzhou, 324000, Zhejiang, China. \\ ${ }^{3}$ Faculty of Information Technology, Macau University of Science \\ and Technology, WaiLong, Taipa, 999078, Macau, China.
}

*Corresponding author(s). E-mail(s): Kaifang@ieee.org; Contributing authors: maokeji@zjut.edu.cn; 843120596@qq.com; 2111912190@zjut.edu.cn; maojiafa@zjut.edu.cn; xiaolong@ieee.org;

\begin{abstract}
The prediction of children's adult height is a common procedure in childhood endocrinology. Through the prediction of children's adult height, it is possible to find abnormalities in children's growth and development. Many jobs in today's society have certain requirements for height, so the accuracy of children adulthood height prediction is important for children. Current methods for predicting adult height of children have some shortcomings such as inaccurate accuracy. To deal with these problems, this paper analyzes the data collected by the Chinese children and adolescents' physical and growth health projects in primary and secondary schools in Zhejiang Province, and proposes a method for predicting adult height based on back propagation neural network (BPNN) with the body composition of children and adolescents as input. Since the BP algorithm has the risk of falling into local optimization, and we propose LSALO-BP model that incorporates the ant lion optimizer (LSALO) into the BP algorithm as location strategy to avoid local optimization. The improvements achieved by the ant lion algorithm are mainly reflected in:
\end{abstract}


improving the ant's walk mode, and enhancing the global search ability of the LSALO algorithm. The comparison experiment of 10 benchmark functions proves the feasibility and effectiveness of the location strategy. The LSALO-BP model is applied to the prediction of adult height of children and adolescents. The experimental results show that compared with other models, the LSALO-BP prediction model has increased the prediction accuracy by $6.67 \% 16.08 \%$ for boys and $4.67 \% 6.6 \%$ for girls, which can more accurately predict the adult height of children and adolescents.

Keywords: adult height prediction of children,location strategy,improved Ant lion optimizer,bp neural network,prediction model

\section{Introduction}

In recent years, the growth and development of children and adolescents have received extensive attention, and especially the height of adulthood has actually affected the future lives of children and adolescents. The National Nutrition Plan issued by the State Council in 2017 clearly stated that the growth retardation rate of Chinese children and adolescents should be kept below $5 \%$ by 2020 to promote the improvement of national physique [1]. The study of children's adult height prediction is mainly used in the selection of athletes [2]. In the selection of athletes, height is a very important morphological index. Some events require athletes to be taller, such as basketball and volleyball; some events require athletes to be shorter, such as weightlifting and diving. This requires coaches to accurately predict their height in adulthood when selecting athletes [3].However, children and adolescents are not all smooth in the process of growth and development, the lack of certain hormones may affect the growth of height.For example, the lack of growth hormone can cause children to be short and unable to reach the standard height of their peers [4]. Therefore, the prediction of the height of children and adolescents in adulthood can not only intervene in the abnormal situations in their growth and development stages in a timely manner, but also have a positive guiding role in the cultivation of life habits in the growth of children. The adult height of children is an important indicator to assess their growth and development, and has always been the focus of attention of the country, doctors and parents [5]. Therefore, the accuracy of the prediction method is very important for the future life of children.

At present, there are many kinds of prediction methods, the most widely used is the back propagation (BP) neural network, which is widely used in various prediction and computing research fields. But the BP neural network has some shortcomings. It is sensitive to weights and thresholds, and it is easy to fall into a local optimal solution. Xiao et al. [6] proposed a BP neural network prediction model based on simulated annealing algorithm, which reduced the possibility of over-fitting of neural network and improved the prediction 
accuracy. On the basis of BP neural network, Hou et al. [7] adopted particle swarm optimization algorithm to optimize it, and put forward PSO-BP prediction model, which improved the accuracy of prediction. In order to overcome the shortcomings of $\mathrm{BP}$ neural network and single genetic algorithm, $\mathrm{Xu}$ et al. [8] proposed an optimized BP neural network based on quantum genetic algorithm (QGA), which optimized the weight and threshold values of BP neural network and improved the convergence speed. Zhang et al. [9] proposed a global accelerated BP (GAD-BP) neural network model, and the initial weight was optimized by Levenberg-Marquardt (LM) algorithm to improve the convergence speed. Teng et al. [10] proposed a BP neural network prediction model based on the improved differential evolution algorithm. The model used the differential evolution algorithm to optimize the weight, reducing the error and improving the accuracy. $\mathrm{Hu}$ et al. [11] proposed an optimized BP neural network based on fuzzy clustering algorithm and generic algorithm.

In terms of children's adult height prediction, computer technology is widely used in this field. Mejía et al. [12] measured children and adolescents' height, weight, ulna, arm span and other physical indicators, and used Bland-Altman method [13] to analyze the difference and consistency between observed height and predicted height. Lello et al. [14] used machine learning methods to construct genomic predictors which use heritable and extremely complex human quantitative characteristics (height, heel bone density and education level). According to the height and weight data of children and adolescents, Shmoish et al. [15] analyzed the feasibility of regression models such as linear, multi-layer perceptron (MLP), decision tree and random forest to predict the adult height of children, and found that the prediction accuracy of random forest regression model was good. Madden et al. [16] improved the prediction formula by measuring ulnar length, which was used to estimate the adult height of children from different ethnic backgrounds. Hyeon et al. [17] proposed a TW3 algorithm using deep learning, which used convolutional neural network to predict the growth level of children's left hand bone, and predicted the adult height of children according to the predicted results.

At present, there are few papers discussing the problem of children's adult height prediction, and there are some drawbacks with the prediction methods, such as low prediction accuracy. In order to accurately predict the adult height of children and adolescents, this paper proposes a children's adult height prediction method based on improved ant lion algorithm (LSALO) and BP algorithm, which is called as LSALO-BP method. Aiming at the problem that the BP algorithm is easy to fall into local optimality, the LSALO algorithm is introduced. The global optimization ability of the algorithm and the error correction ability of the BP algorithm are used to improve the performance of the neural network. Overall, main contributions are as follows.

(1) A data set of heights of children and adolescents in some cities of Zhejiang Province of China was constructed, including Hangzhou, Shaoxing, Wenzhou and other cities. There are 1096 data items collected, and the content of the data includes height, weight, age, bone age, and so on. 
(2) The LSALO-BP algorithm is adopted. Aiming at the problem that the $\mathrm{BP}$ algorithm is easy to fall into local optimality, an ant lion algorithm based on location strategy is proposed to optimize the BP algorithm. The main improvement of the algorithm is to improve the way of ants and antlions, and improve the global search ability.

(3) Comparing the LSALO-BP hybrid model with other models, the accuracy of boys is increased by $6.67 \% 16.08 \%$, and the accuracy of girls is increased by $4.67 \% 6.6 \%$.

\section{Problem formulation}

\subsection{Children adulthood height}

Nowadays, many industries have rigid requirements for height, for example, pilots or models. If children can predict their adult height as early as possible, they can avoid many detours in the growth process. In the diagnosis of growth and development of children and adolescents, the dynamic change of height in adulthood is one of the criteria for judging whether the development is abnormal.

Height prediction in adulthood is a long process and requires continuous observation of a large sample. The growth trend of children and adolescents with abnormal development is often more difficult to determine than normal children and adolescents. The easiest way to predict the adult height of children is to use the height of the parents to predict by using the genetic height formula. The genetic formula will change as the growth samples of children and adolescents change, and when children and adolescents develop physical diseases during their growth, the formula will become inapplicable. At present, most doctors use the method of height prediction based on bone age, combined with their own experience for diagnosis and prediction, but this method is affected by various subjective factors and is prone to misjudgment. With the progress of society, the physical fitness of children has also been strengthened, and many prediction methods need to be changed.

\subsection{Children adulthood height}

The neural network is composed of an input layer, a hidden layer, and an output layer, and the application scenarios are very wide. A typical neural network is shown in Figure 1.

There are two points in the selection criterion of neural network input variables [18]. One is that the input variables should be closely related to the predicted objects, and the other is that there cannot be a strong linear relationship between the input variables. Based on this criterion, the neural network prediction model selects children's height, age and bone age as input values, The expression equation is as follows:

$$
X_{i}=\left(X_{(\text {age }) i}, X_{(\text {boneage })}, X_{(\text {height }) i}\right)
$$




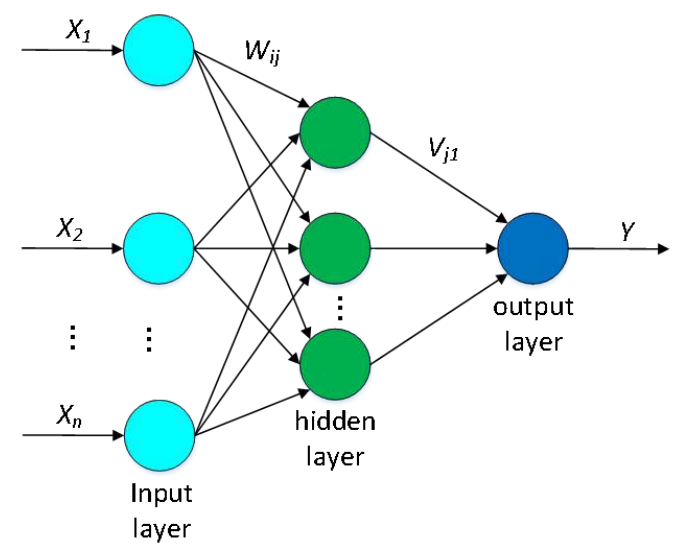

Fig. 1 A traditional neural network

Where $X_{i}$ represents the input value, $X_{(a g e) i}$ is the detection age, $X_{(\text {boneage })}$ is the current estimated bone age, $X_{(\text {height }) i}$ is the current height. The output layer uses the child's adult height as the output value. The error function is defined as follows:

$$
F_{M S E}=\frac{1}{n} \sum_{i=1}^{n}\left(Y_{i}-{ }_{-} Y_{i}\right)^{2}
$$

Where $n$ is the total number of samples, $Y_{i}$ is the $i$-th actual value of the sample, and $Y_{i}$ is the $i$-th predicted value of the neural network.

We know that the BP algorithm uses the standard gradient descent method based on the decreasing function of error and performance index [19], which is a local search optimization method. The disadvantage of this method is that it is easy to fall into the local optimal solution. In neural networks, we often use the BP algorithm to optimize the weights of the neural network, and the neural network is extremely sensitive to the initialization weights. Training the network with different initialization weights often results in different results. If the network falls into local optimum, it will lead to unsatisfactory training results. In order to overcome this problem, this paper will combine the improved Ant Lion Optimization Algorithm (LSALO) with the BP algorithm to make up for the deficiencies of the BP algorithm.

\subsection{Ant Lion optimizer}

Ant Lion Optimizer (ALO) is a swarm intelligence optimization algorithm, which was proposed by Seyedali in 2015 [20]. This algorithm searches the global optimal solution by simulating the behavior of the ant lion in nature to capture the ants. Ant lion optimizer includes the following roles: elite ant lion, ant lion and ant. Ant represents an attempted solution and it can walk randomly within the range and gradually approach the ant lion to find the optimal position within the range. Ant lion represents a local optimal solution. 
When the ants walking around the ant lion find a better position than the ant lion, they will be eaten by the ant lion, and the current position of the ant will be replaced by the ant lion. The elite ant lion represents the global optimal solution. After each update, the best ant lion will be selected from all the ant lions as the elite ant lion. Through such continuous iterations, a more accurate global optimal solution is finally obtained.

The process of ants randomly walking can be seen as the process of searching for feasible fields in the search space. The mathematical expression is shown by equation (3):

$$
X(t)=[0, \text { cumsum }[2 r(1)-1, \text { cumsum }[2 r(t)-1], \ldots, \text { cumsum }[2 r(T)-1]]]
$$

Where cumsum represents the cumulative sum of positions, $t$ represents the current number of iterations, $T$ represents the maximum number of iterations, and $r(t)$ is a random function 0 or 1 .

$$
r(t)= \begin{cases}1, & m>0.5 \\ 0, & m \leq 0.5\end{cases}
$$

where $m$ is a random number evenly distributed in the range $[0,1]$. However, the range of ants' walk is not unlimited, and through equation (5), ants can only move randomly in the search space.

$$
X_{i}^{t}=\frac{\left.\left(X_{i}^{t}-a_{i}\right)\left(n_{i}-c_{i}^{t}\right)\right)}{d_{i}^{t}-a_{i}}+c_{i}
$$

where $b_{i}$ and $a_{i}$ are the upper bound and the lower bound of the $i$-th variable respectively, $d_{i}^{t}$ and $c_{i}^{t}$ are the upper and lower bounds of the $i$-th variable at the $t$-th iteration. The ants are easily affected by traps during the random walk, and gradually approach the ant lion, so the definitions of $c_{i}^{t}$ and $d_{i}^{t}$ are shown in equation (6) and equation (7):

$$
\begin{aligned}
& c_{i}^{t}=A l_{j}^{t}+c^{t} \\
& d_{i}^{t}=A l_{j}^{t}+d^{t}
\end{aligned}
$$

where $d^{t}$ and $c^{t}$ are the upper and lower bounds of the value range of all variables at the $t$-th iteration, a is the position of the $j$-th ant lion at the $t$-th generation.

The fitness function can be used to judge the position of the ants. The higher the fitness, the better the ant's location. In each iteration, if there is a more adaptable ant within the hunting range of the ant lion, the ant lion will capture and eat it, and the original position of the ant is replaced by the ant lion. The mathematical expression is shown in equation (8):

$$
A l_{j}^{t}=A n t_{i}^{t} \quad \text { if } \quad f\left(A n t_{i}^{t}\right)>f\left(A l_{i}^{t}\right)
$$


where $A l_{j}^{t}$ is the position of the $i$-th ant lion in the $t$-th generation and $A n t_{i}^{t}$ is the position of the $j$-th ant in the $t$-th generation. $f$ is the fitness function.

After each iteration, one of the most adaptable ants is selected from all ant lions as an elite ant lion. When the number of iterations reaches the maximum number of iterations or the error is within, the search is stopped, and the elite ant lion at this time represents the global optimal solution within the scope of the solution space.

\section{Proposed LSALO-BP method}

\subsection{Improved ant lion optimizer}

1) Ant lion replacement method. In the original ALO algorithm, the ant walks randomly around the ant-lion. If the ant-lion is not in a good position, the ant's optimization process is equivalent to searching for the local optimal solution in a local scope instead of obtaining the global optimal solution. Therefore, in order to reduce the probability of this happening, the following replacement operations are performed on ant lion at each iteration:

Step 1: After an iteration is completed, randomly initialize the same number of ant lions.

Step 2: Calculate the fitness value of ant lion, and sort the original group of ant lions and the new group of ant lions, and the ant lions with higher fitness value are ranked in front.

Step 3: Use the greedy selection mechanism to select the same number of ant lions with higher fitness.

The mathematical expression is as follows, if the fitness of $F A_{i}^{t}$ is lower than $F B_{i}^{t}$, then:

$$
\begin{aligned}
& F A_{i}^{t}=F B_{i}^{t} \\
& P A_{i}^{t}=P B_{i}^{t}
\end{aligned}
$$

where $F A_{i}^{t}$ is the fitness value of the original group, and $F B_{i}^{t}$ is the fitness value of the new group, $P A_{i}^{t}$ shows the position of the original group, and $P B_{i}^{t}$ shows the position of the new group. $i$ is the $i$-th ant lion of the original group, and $j$ is the $j$-th ant lion of the new group. $t$ refers to the current number of iterations.

2) Ants walk way. Each new replacement ant lion has a high adaptability, but there will be cases where it is not selected by roulette, which causes ant lion replacement to become a meaningless action and cannot play its role. Therefore, as long as it is judged that there is a replacement of new ant lions, the ants will walk around randomly to ensure that each new ant lion has its meaning of existence. The elite ant lion is the global optimal solution selected after each iteration. It will greatly affect the ants' random walk movements, so when iterating, change the ant's walking route, and let the ants walk randomly around the roulette chosen ant lion, the new replacement ant lion, and the elite ant lion. In the ant lion optimization algorithm, the step size of the early 
ant walk is larger, and the step size is smaller the later, until it converges to a certain position. Therefore, the step size adjustment function $\frac{1}{e^{\log t}}$ is introduced to adjust the process of ants' random walk. The mathematical expression is as follows:

$$
\begin{aligned}
& R A=\operatorname{Rand}_{A}^{t}=\operatorname{rand}(-1,1) * \frac{1}{e^{\log t}} \\
& R E=\operatorname{Rand}_{E}^{t}=\operatorname{rand}(-1,1) * \frac{1}{e^{\log t}} \\
& R C=\operatorname{Rand}_{C}^{t}=\operatorname{rand}(-1,1) * \frac{1}{e^{\log t}}
\end{aligned}
$$

where $\operatorname{Rand}_{A}^{t}$ means randomly walking around the roulette chosen ant lion in the $t$ iteration, $\operatorname{Rand}_{E}^{t}$ means randomly walking around the elite ant lion in the $t$ iteration, means randomly walking around the new replacement ant lion in the $t$ iteration, the function image of the step adjustment function $\frac{1}{e^{\log t}}$ is shown below:

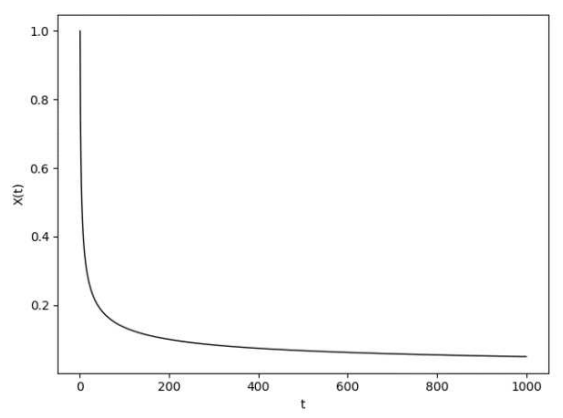

Fig. $2 \frac{1}{e^{\log t}}$ function image

When each iteration is completed, the mathematical expression of the final position of the ant is as follows:

$$
A T_{i}^{t}=x \times R A+y \times R E+z \times R C
$$

where $A T_{i}^{t}$ is the position of the $i$-th ant in the $t$ iteration, the sum of $x$, $y$, and $z$ is 1 , and the default value is $\frac{1}{3}$ each, and the specific value can be adjusted according to the actual situation.

3) Ant replacement method. Suppose that there are $q$ ant lions replaced. After the process of ants' walk, $q$ new ants will appear. These ants get the final position according to formula (14). These new ants will inevitably have a better position than the original ants. The better the ant's position and the higher the fitness value, the easier it will be "eaten" by the ant lion. Therefore, the position of the ant determines to a certain extent where the optimal solution appears. In order to enhance the optimization ability of the 
LSALO algorithm, the ants with low fitness are replaced. The mathematical expression is as follows. If the fitness of $A N_{p}$ is higher than $A O_{\text {min }}^{t}$, then:

$$
\begin{aligned}
& A O_{\text {min }}^{t}=A N_{p} \\
& P O_{\text {min }}^{t}=P N_{p}
\end{aligned}
$$

where $A O_{\min }^{t}$ represents the fitness value of the ant with the lowest fitness in the $t$-th iteration, and $A N_{p}$ represents the fitness value of the $p$-th ant among the new ants. $P O_{\min }^{t}$ shows the location of the ant with the lowest fitness in iteration $t$, and $P N_{p}$ shows the location of the $p$-th ant among the new ants.

\subsection{LSALO-BP network model}

As shown in Figure 3, the algorithm consists of two parts, the LSALO part on the left and the BP neural network training part on the right. The specific steps are as follows:

Step 1: Initialize weights and other parameters;

Step 2: Calculate and record the fitness value of each ant lion;

Step 3: Find the highest fitness value as the elite ant lion, and perform an iteration;

Step 4: Determine whether the stop iteration condition is satisfied, if it is satisfied, end the loop, otherwise skip to the next step;

Step 5: Use equations (9) and (10) to replace the ant lion with lower fitness value, and use the greedy selection mechanism to select the ant lion;

Step 6: Ants walk randomly according to equation (14) and update their position and fitness value;

Step 7: Use equations (15) and (16) to replace ants with low fitness values. Jump to Step 3.

\section{Results}

Data collection uses DR testing equipment, body composition analyzer and other equipment. The DR detection equipment is composed of high-voltage emitters, amorphous silicon flat panel detectors and other devices. The bone age film taken has the advantages of large gray scale dynamic range and high density resolution. The collection of body composition information is applicable to the body composition analyzer model GAIA KIKO. The instrument is simple to operate, with fast test speed and high accuracy. information about bone age, body composition, etc. is collected through these devices. Taking the students of each school as the collection object and taking the class as the unit, the bone age film and the body composition (such as height, weight, BMI, etc.) are measured in sequence for the students. Export and organize the obtained data information from the test equipment and save it in the corresponding database. 
LSALO

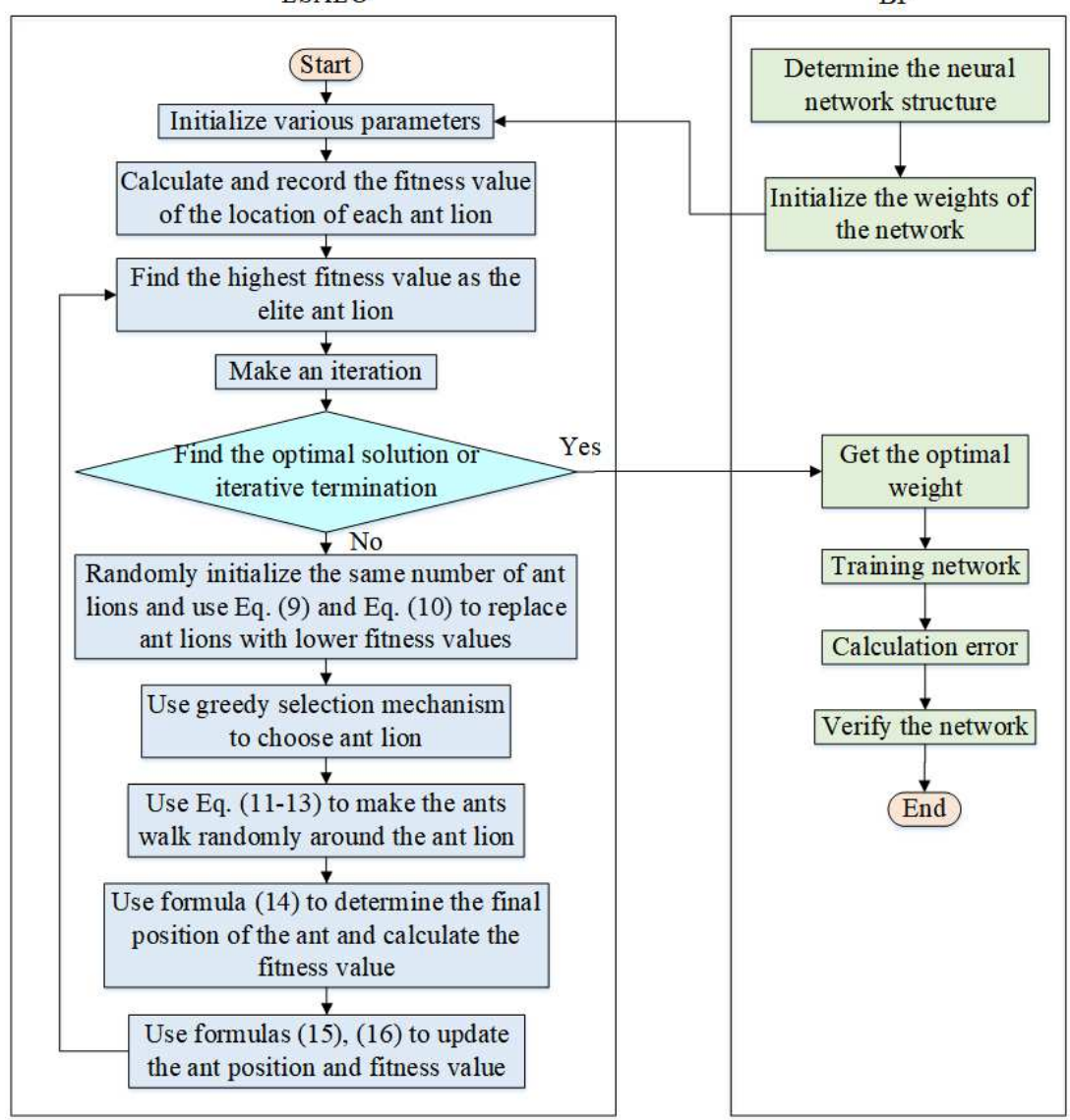

Fig. 3 LSALO-BP algorithm flowchar

\subsection{Data source and analysis}

The data set used in this article comes from primary and secondary schools in Zhejiang Province. We obtained the basic information of the children and confirmed the height of the tester in adulthood through a return visit, a total of 1096 people, including 647 boys and 449 girls. The division of training set and test set is shown in Table 1.

Table 1 The division of training set and test set

\begin{tabular}{lccc}
\hline Gender & Total People & Training Set & Test Set \\
\hline Boy & 647 & 546 & 128 \\
Girl & 449 & 355 & 94 \\
\hline
\end{tabular}


In the return visit data, there are 128 boys and 94 girls. Table 2 and Table 3 show the structure of part of the return visit data for boys and girls, including the child's name, date of return visit, bone age, age, and height. The samples taken from various schools in Zhejiang Province ensure that the health of the data is better than the data collected from the hospital, and the detection data of the urban and rural schools are combined and processed to obtain a general average level.

Table 2 Basic information of boys' return visit

\begin{tabular}{lcccl}
\hline Name & Return visit date & Age & Bone age & height \\
\hline Fu** $^{* * *}$ & $2018 / 7 / 26$ & 11.8 & 12.8 & 144.7 \\
Chen* & $2018 / 7 / 26$ & 13.6 & 14.7 & 165 \\
Zhou** & $2018 / 7 / 26$ & 14.9 & 16 & 194.5 \\
Xie** & $2018 / 7 / 26$ & 12.2 & 12.6 & 152.2 \\
Zhu** & $2018 / 7 / 26$ & 13.2 & 15.9 & 156.5 \\
Liu** $_{\text {Ye** }}^{2018 / 7 / 26}$ & 12.6 & 13.1 & 148.5 \\
Wang* & $2018 / 7 / 26$ & 13.9 & 12.8 & 158.8 \\
.. & $2018 / 7 / 27$ & 13.9 & 13.3 & 152.5 \\
Chai** & $2018 / 8 / 15$ & 13 & 14.9 & 164.5 \\
Zhang** & $2018 / 8 / 15$ & 13.7 & 13.4 & 164 \\
\hline
\end{tabular}

Table 3 Basic information of girls' return visit

\begin{tabular}{lcccl}
\hline Name & Return visit date & Age & Bone age & height \\
\hline Tao** & $2018 / 7 / 21$ & 13.6 & 12 & 155.8 \\
Jin** & $2018 / 7 / 21$ & 11.9 & 13 & 152.4 \\
Zhang** & $2018 / 7 / 21$ & 13 & 13.1 & 165 \\
Xie* & $2018 / 7 / 21$ & 11.8 & 12.3 & 150.5 \\
Wang** & $2018 / 7 / 21$ & 13.4 & 14.7 & 157 \\
Hu** & $2018 / 7 / 18$ & 11.4 & 11.8 & 148 \\
Wang** & $2018 / 7 / 18$ & 11.7 & 11.3 & 147 \\
Kong* & $2018 / 7 / 18$ & 11.8 & 11.5 & 144.2 \\
$\ldots$ & $\ldots .$. & $\ldots$ & $\ldots$ & $\ldots$ \\
Zhou** & $2018 / 8 / 10$ & 11.6 & 11.5 & 142.4 \\
Xi** & $2018 / 8 / 11$ & 11.6 & 12.3 & 152.8 \\
\hline
\end{tabular}

Through the collation and analysis of the sample data of the return visit, the basic distribution of the number of ages in the sample is shown in Figures 4 and 5. Taking 6 years old as an example, it means the number of people between 6 years old and 0 months to 6 years old and 12 months. In the collected data samples, the male and female samples are relatively evenly distributed. It can be seen from the comparison of the two pictures that girls start to develop earlier than boys. 


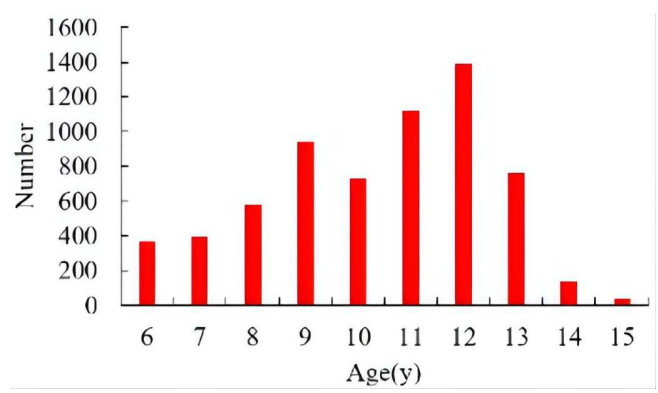

Fig. 4 Distribution of boys' age

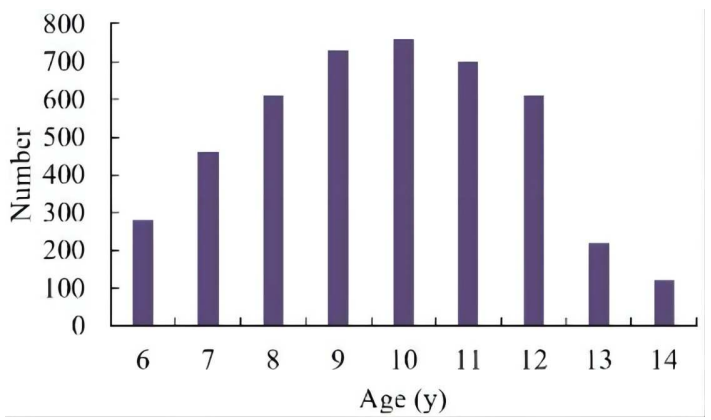

Fig. 5 Distribution of girls' age

The distribution of bone age in the sample is shown in Figures 6 and 7. According to the relationship between bone age and age, children's development can be divided into three types, delayed development, normal development and early development. Delayed development means that the bone age is more than one year younger than actual age, normal development means that the bone age and actual age are within 1 year of each other, and early development means that the bone age is more than one year older than the actual age. The sample includes children with the above three developmental conditions, so we have adequate data to fit.

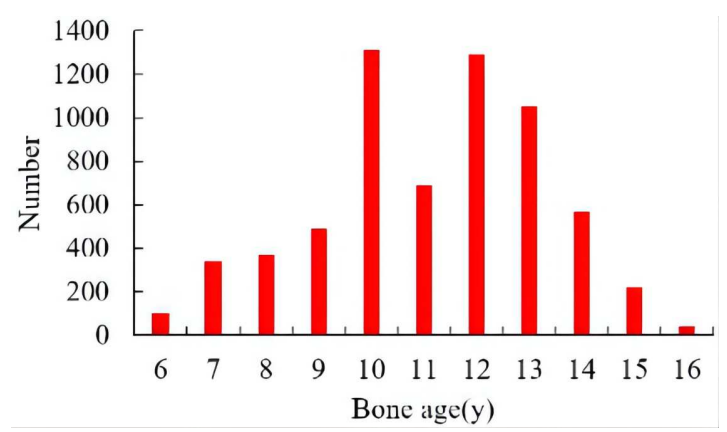

Fig. 6 Distribution of bone age of boys 


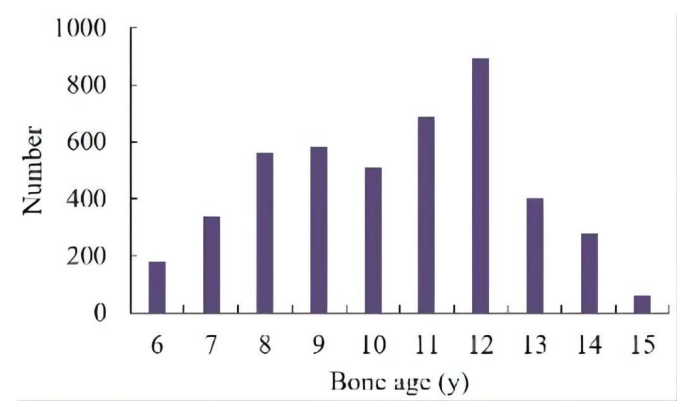

Fig. 7 Distribution of bone age of girls

\subsection{Benchmark function evaluation}

In order to verify the effectiveness of the location strategy, this paper selects 10 benchmark functions for comparative experiments of the algorithm, as shown in Table 4. $f_{1} f_{4}$ are unimodal functions, $f_{5} f_{7}$ are multimodal functions, $f_{8} f_{1} 0$ are low and fixed dimensional multimodal functions. Objects of comparison are the original ALO algorithm and the OB-L-ALO algorithm proposed in [21].

Table 4 Benchmark functions

Function

Search Range Optimum Value

$\begin{array}{lrr}f_{1}(x)=\sum_{i=1}^{n} x_{i}^{2} & {[-100,100]} & 0 \\ f_{2}(x)=\sum_{i=1}^{n}\left|x_{i}\right|+\prod_{i=1}^{n}\left|x_{i}\right| & {[-10,10]} & 0 \\ f_{3}(x)=\max _{1 \leq i \leq n}\left\{\left|x_{i}\right|\right\} & {[-100,100]} & 0 \\ f_{4}(x)=\sum_{i=1}^{n}\left(\left[x_{i}+0.5\right]\right)^{2} & {[-100,100]} & 0 \\ f_{5}(x)=\sum_{i=1}^{n}-x_{i} \sin \left(\sqrt{\left|x_{i}\right|}\right) & {[-500,500]} & -418.9829^{*} \mathrm{Dim} \\ f_{6}(x) & {[-32,32]} & 0 \\ \exp \left(\frac{1}{n} \cos \left(2 \pi x_{i}\right)\right)+20+e & & \\ f_{7}(x)=0.1\left\{\sin ^{2}\left(3 \pi x_{1}\right)+\sum_{i=1}^{n}\left(x_{i}-1\right)^{2}\left[1+\sin ^{2}\left(3 \pi x_{i}+\right.\right.\right. & {[-50,50]} & \\ \left.1)+\left(x_{n}-1\right)^{2}\left[1+\sin ^{2}\left(2 \pi x_{n}\right)\right]\right\}+\sum_{i=1}^{n} u\left(x_{i}, 5,100,4\right) & & \\ f_{8}(x)=\left[\frac{1}{500} \sum_{j=1}^{25}\left(j+1+\sum_{i=0}^{1}\left(x_{i}-a_{i j}\right)^{6}\right)^{-1}\right] & {[-65.54,65.54]} & 0.998 \\ \left.f_{9}(x)=\sum_{i=0}^{10}\left(a_{i}-\frac{x_{0}\left(b_{i}^{2}+b_{i} x_{1}\right)}{b_{i}^{2}+b_{i} x_{2}+x_{3}}\right)^{2}\right) & {[-5,5]} & \\ f_{10}(x)=4 X_{0}^{2}-2.1 x_{0}^{4}+\frac{1}{3} x_{0}^{6}+x_{0} x_{1} & \left.-\sum_{i=1}^{2}\right) & 0.0003075\end{array}$


In order to reflect the fairness of this comparison experiment, the three algorithms use the same parameter settings, that is, the population size $\mathrm{N}=30$, the maximum number of iterations $\mathrm{T}=1000$, and different algorithms are run independently for each benchmark function 30 times, and the average value of the experimental results is compared to reduce the impact of randomness. Table 5 shows the experimental results of the ALO algorithm, OB-L-ALO algorithm and LSALO algorithm, and Figure 9 shows the comparison of the iterative convergence curves of the ALO algorithm and the LSALO algorithm.

Table 5 Comparison of optimization results of benchmark functions by different algorithms

\begin{tabular}{|c|c|c|c|}
\hline Function & Algorithm & Average & Standard Deviation \\
\hline & ALO & $4.01 \mathrm{E}-05$ & $4.49 \mathrm{E}-05$ \\
\hline \multirow{3}{*}{$f 1$} & OB-L-ALO & $1.01 \mathrm{E}-09$ & 4.63E-09 \\
\hline & LSALO & $1.49 \mathrm{E}-11$ & $4.92 \mathrm{E}-12$ \\
\hline & ALO & $4.97 \mathrm{E}-06$ & $3.23 \mathrm{E}-06$ \\
\hline \multirow[t]{3}{*}{$f 2$} & OB-L-ALO & $5.98 \mathrm{E}-05$ & $4.26 \mathrm{E}-05$ \\
\hline & LSALO & 5.13E-06 & $7.25 \mathrm{E}-06$ \\
\hline & ALO & $6.79 \mathrm{E}-05$ & $3.36 \mathrm{E}-05$ \\
\hline \multirow[t]{3}{*}{$f 3$} & OB-L-ALO & $1.09 \mathrm{E}-05$ & $2.29 \mathrm{E}-05$ \\
\hline & LSALO & $4.14 \mathrm{E}-06$ & $1.56 \mathrm{E}-06$ \\
\hline & ALO & $5.14 \mathrm{E}-06$ & 3.23E-06 \\
\hline \multirow[t]{3}{*}{$f 4$} & OB-L-ALO & $3.23 \mathrm{E}-05$ & $1.84 \mathrm{E}-05$ \\
\hline & LSALO & $1.05 \mathrm{E}-08$ & $6.81 \mathrm{E}-09$ \\
\hline & ALO & $-5.90 \mathrm{E}+03$ & $8.87 \mathrm{E}+02$ \\
\hline \multirow[t]{3}{*}{$f 5$} & OB-L-ALO & $-5.98 \mathrm{E}+03$ & $1.71 \mathrm{E}+03$ \\
\hline & LSALO & $-7.80 E+03$ & $1.40 \mathrm{E}+03$ \\
\hline & ALO & $2.27 \mathrm{E}-05$ & $3.72 \mathrm{E}-06$ \\
\hline \multirow[t]{3}{*}{$f 6$} & OB-L-ALO & $2.10 \mathrm{E}-05$ & $1.46 \mathrm{E}-05$ \\
\hline & LSALO & $1.99 \mathrm{E}-06$ & 2.77E-07 \\
\hline & ALO & $1.47 \mathrm{E}-03$ & 3.73E-03 \\
\hline \multirow{3}{*}{$f 7$} & OB-L-ALO & $6.98 \mathrm{E}-06$ & $5.33 \mathrm{E}-06$ \\
\hline & LSALO & $4.98 \mathrm{E}-09$ & $4.64 \mathrm{E}-09$ \\
\hline & ALO & $1.30 \mathrm{E}+00$ & $0.46 \mathrm{E}+00$ \\
\hline \multirow[t]{3}{*}{$f 8$} & OB-L-ALO & $1.82 \mathrm{E}+00$ & $1.08 \mathrm{E}+00$ \\
\hline & LSALO & 9.98E-01 & $3.33 \mathrm{E}-16$ \\
\hline & ALO & 7.19E-04 & $1.05 \mathrm{E}-04$ \\
\hline \multirow[t]{3}{*}{$f 9$} & OB-L-ALO & $8.21 \mathrm{E}-04$ & $2.05 \mathrm{E}-04$ \\
\hline & LSALO & $7.05 \mathrm{E}-04$ & $9.11 \mathrm{E}-05$ \\
\hline & ALO & $-1.03 \mathrm{E}+00$ & $4.00 \mathrm{E}-14$ \\
\hline \multirow{2}{*}{$f 10$} & OB-L-ALO & $-1.03 \mathrm{E}+00$ & $6.78 \mathrm{E}-16$ \\
\hline & LSALO & $-1.03 E+00$ & $2.50 \mathrm{E}-15$ \\
\hline
\end{tabular}

In Table 5 and Figure 8, the LSALO algorithm has achieved better experimental results than other algorithms. For unimodal functions $f_{3}$ and $f_{4}$, the average experimental results of LSALO algorithm are superior to ALO algorithm and OB-L-alo algorithm, with an increase of 1-5 orders of magnitude and a smaller standard variance, indicating that the algorithm has strong optimization ability and good stability. By comparing the convergence curves of the functions $f_{3}$ and $f_{4}$, the LSALO algorithm has higher convergence accuracy. 


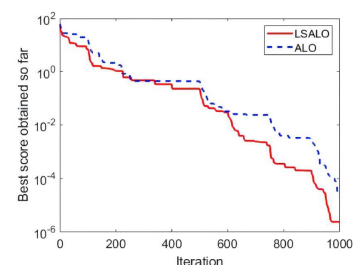

(a) $f_{3}$

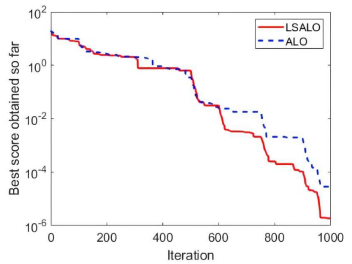

(d) $f_{6}$

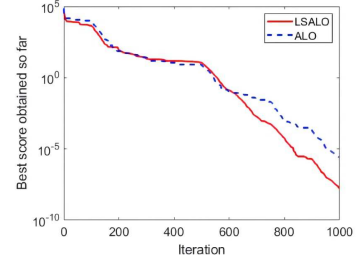

(b) $f_{4}$

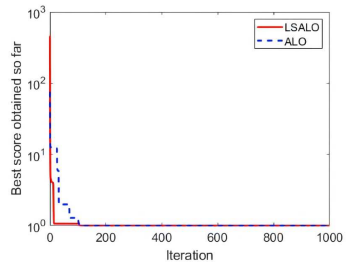

(e) $f_{8}$

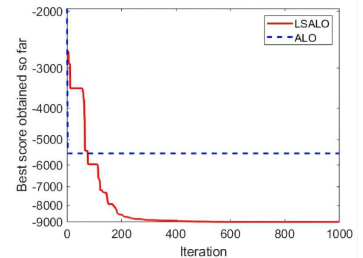

(c) $f_{5}$

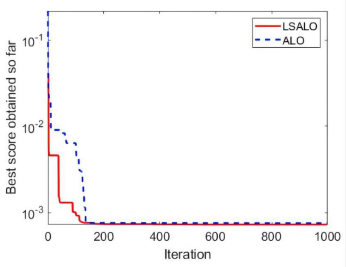

(f) $f_{9}$

Fig. 8 Iteration curve of benchmark functions

For the multimodal function $f_{5}$, the ALO algorithm falls into local optimality with less than 100 iterations, resulting in an early end to the algorithm optimization process. The position strategy of LSALO algorithm makes it not easy to fall into the local optimum and the global search ability is stronger. For the low and fixed dimensional multimodal functions $f_{8}$ and $f_{10}$, the average value of the results of the LSALO algorithm has reached the theoretical optimal value, and the optimization rate has reached $100 \%$.

In summary, according to the optimization results of the benchmark functions, it can be seen that LSALO is superior to the ALO algorithm and the OB-L-ALO algorithm in terms of global optimization ability and convergence accuracy. It shows that the algorithm is feasible and effective, and can be further applied to the weight optimization problem of BP neural network.

\subsection{Analysis of children's adult height prediction results}

In this experiment, the height, bone age, and age of children at the time of testing were selected as input, and adult height was output. The evaluation is separately conducted for boys and girls. Body composition information such as bone age and age is not a simple linear relationship with adult height, and each child's growth and development are different, resulting in a variety of input characteristics. This experiment uses a double hidden layer neural network. A large number of experiments show that when the number of neurons in the first hidden layer is twice the number of input values, and the number of neurons in the second hidden layer is the same as the number of input values, the BP neural network has the best training effect. Therefore, it can be determined that the structure of the neural network is 3-6-3-1.

This experiment uses the LSALO algorithm, PSO algorithm [? ] and GA algorithm [? ] to optimize the BP neural network to form the LSALO-BP network model, GA-BP network model and PSO-BP network model. The three 
models are compared with the original BP network model and the BayleyPinneau method, and the samples are predicted and analyzed.

This paper selects whether the difference between the true value and the predicted value is within $\pm 2 \mathrm{~cm}$ as the basis for accuracy judgment. Each model is run independently for 10 times, and the average value is taken as the final result. The analysis results of this experiment are shown in Table 6 and Table 7. Table 6 shows the analysis results of male height prediction, and Table 7 shows the analysis results of female height prediction. It can be seen from Table 6 that LSALO-BP model's prediction results for boys are superior to other prediction models in terms of accuracy and standard deviation, with an accuracy rate of $86.67 \%$ and a standard deviation of $1.176 \mathrm{~cm}$. Compared with BP neural network, the accuracy rate is even improved by $16.08 \%$. From Table 7 , we can see that the prediction results of LSALO-BP model for girls are still better than other prediction models in terms of accuracy and standard deviation, with an accuracy rate of $85.32 \%$ and a standard deviation of $1.615 \mathrm{~cm}$.

Table 6 Adult height prediction analysis of boys

\begin{tabular}{ccc}
\hline Prediction model & Accuracy & Standard deviation \\
\hline LSALO-BP Model & $86.67 \%$ & 1.176 \\
PSO-BP Model & $77.84 \%$ & 1.467 \\
GA-BP Model & $80.00 \%$ & 1.659 \\
BP Model & $70.59 \%$ & 1.464 \\
Bayley-Pinneau & $70.59 \%$ & 3.343 \\
\hline
\end{tabular}

Table 7 Adult height prediction analysis of girls

\begin{tabular}{ccc}
\hline Prediction model & Accuracy & Standard deviation \\
\hline LSALO-BP Model & $85.32 \%$ & 1.615 \\
PSO-BP Model & $79.57 \%$ & 1.775 \\
GA-BP Model & $79.36 \%$ & 1.659 \\
BP Model & $78.72 \%$ & 1.902 \\
Bayley-Pinneau & $80.85 \%$ & 2.404 \\
\hline
\end{tabular}

The error analysis results of this experiment are shown in figure 9, and boxplot is adopted to display. Boxplots can intuitively determine the discrete distribution of errors, understand the distribution of errors, and identify outliers in errors. The range of error is expressed by the vertical distance between the minimum and maximum values, and the interquartile range (IQR) of the error is expressed by the height of the box. Figure (a) is the error analysis result of boys, and figure (b) is the error analysis result of girls. As can be seen 


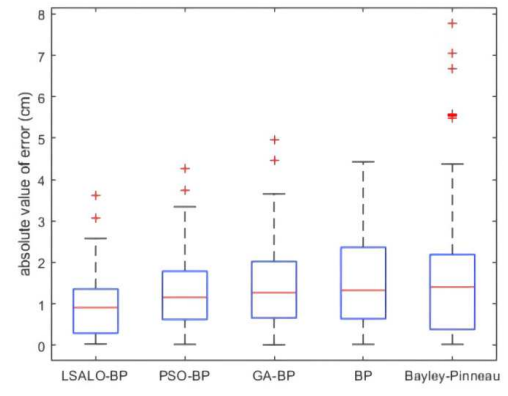

(a) male error distribution chart

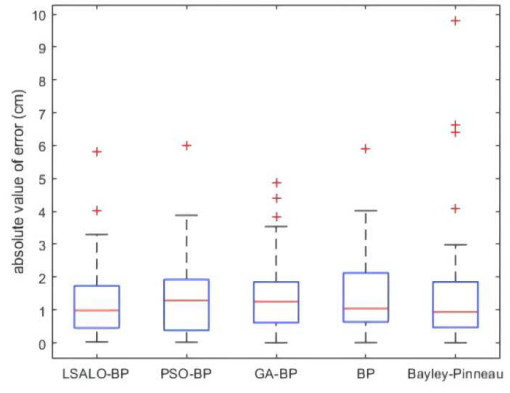

(b) female error distribution chart

Fig. 9 Iteration curve of benchmark functions

from figure (a), the quartile interval and median of LSALO-BP model prediction error are better than other prediction models. As can be seen from figure (b), the quartile interval and median of LSALO-BP model prediction error are similar to the Bayley-Pinneau method, but the number of outliers is less than the Bayley-Pinneau method, indicating that LSALO-BP model has better stability. Based on the above analysis, we can conclude that the performance of the LSALO-BP model prediction model is superior to other prediction models.

\section{Conclusion}

Aiming at the defect that the traditional BP neural network has fallen into local optimality, we propose a location strategy ant lion optimizer (LSALO) to improve the BP network. By changing the way ants walk and the replacement methods of ants and ant lions, the global search ability and local optimization ability are enhanced, and the problem that the algorithm is prone to fall into local optimization is improved. Through the optimization solution of different benchmark functions, LSALO is compared with the original ant lion algorithm and its improved algorithm, and the effectiveness of the improved strategy is verified. The LSALO algorithm combined with the BP neural network is used to predict the adult height of children, and four different prediction models are selected for comparison. The experimental results show that the prediction accuracy of the LSALO-BP model is higher and the error fluctuation is smaller, which further enhances the performance of the BP neural network and can accurately predict the adult height of children.

Acknowledgments. This work was supported in part by the National Natural Science Foundation of China (Grant No.62072410,61876168), the Basic Public Welfare Research Project of Zhejiang Province (Grant No.LGG22F020014) and Quzhou Science and Technology Projects(Grant No.2020K19).

Author Contributions. Keji Mao:concept propose,funding support,draft review and algorithm design.Lijian Chen:coding,experimental evaluation and 
manuscript draft writing. Xinben Fan and Jiafa Mao:experiments and analysis. Xiaolong Zhou:funding suport and draft review. Kai Fang:experiment guidance.

\section{Declarations}

Conflict of interest. All authors declare that they have no conflict of interest.

Ethical approval. This article does not contain any studies with human participants or animals performed by any of the authors.Informed consent was obtained from all individual participants included in the study.

Data availability statement:. The raw/processed data required to reproduce these findings cannot be shared at this time as the data also forms part of an ongoing study.

\section{References}

[1] Chinese Nutrition Society: National nutrition plan (2017-2030). Nutr. 39(4), 315-320 (2017)

[2] Xiong, Q.L., Hou, W.S., Xiao, N., Chen, Y.X., Yao, J., Zheng, X.L., Liu, Y., Wu, X.Y.: Motor skill development alters kinematics and co-activation between flexors and extensors of limbs in human infant crawling. IEEE Transactions on Neural Systems and Rehabilitation Engineering 26(4), 780-787 (2018)

[3] J. Liu, Y.H. S. Chen, Wang, C.: An intelligent identification model for the selection of elite rowers by incorporating internet-of-things technology. IEEE Access 8(4), 31234-31244 (2020)

[4] Ashraf, T.S., Ahmed, E., Maya, I., Celine, J., Vincenzo, D.S.: Responses to growth hormone (gh) therapy in short children with normal gh secretion and no bone age delay: an analysis of potential factors affecting their response to rhgh therapy. a controlled study. Acta bio-medica : Atenei Parmensis 90(8-S), 43-51 (2019)

[5] W. Di, F.G., Chunxiu, G.: Analysis of the therapeutic effect of growth hormone therapy in children with idiopathic short stature followed up to near adult life height. Cap. Univ. Med Sci. 39(1), 92-97 (2018)

[6] Y. Xiao, Y.Z. J. Li, Li, Q.: User behavior prediction of social hotspots based on multimessage interaction and neural network. IEEE Trans. Comput. Soc. Syst. 7(2), 536-545 (2020)

[7] Hou, C., Yu, X., Cao, Y., Lai, C., Cao, Y.: Prediction of synchronous closing time of permanent magnetic actuator for vacuum circuit breaker based 
on pso-bp. IEEE Transactions on Dielectrics and Electrical Insulation 24(6), 3321-3326 (2017)

[8] Xu, L., Zhao, S., Li, N., Gao, Q., Wang, T., Xue, W.: Application of qga-bp for fault detection of liquid rocket engines. IEEE Transactions on Aerospace and Electronic Systems 55(5), 2464-2472 (2019)

[9] Zhang, D., Hu, G., Lu, J., Yin, X., Ren, Q.: Short-term load forecasting based on gad-bp neural network. In: 2019 IEEE 3rd Advanced Information Management, Communicates, Electronic and Automation Control Conference (IMCEC) (2019)

[10] Teng, Y., Huang, X., Ye, S., Li, Y.: Prediction of particulate matter concentration in chengdu based on improved differential evolution algorithm and bp neural network model. In: 2018 IEEE 3rd International Conference on Cloud Computing and Big Data Analysis (ICCCBDA), pp. 100-106 (2018). IEEE

[11] Hu, H., Zhai, X., Feng, J., Guan, X.: Prediction method of crude oil production based on fcm_ga_bp neural network. In: 2018 IEEE 9th International Conference on Software Engineering and Service Science (ICSESS), pp. 267-270 (2018). IEEE

[12] Hu, H., Zhai, X., Feng, J., Guan, X.: Prediction method of crude oil production based on fcm_ga_bp neural network. In: 2018 IEEE 9th International Conference on Software Engineering and Service Science (ICSESS), pp. 267-270 (2018). IEEE

[13] Giavarina, D.: Understanding bland altman analysis. Biochemia Medica 25(2), 141-151 (2015)

[14] Lello, L., Avery, S.G., Tellier, L., Vazquez, A.I., Hsu, S.: Accurate genomic prediction of human height. Genetics 210(2) (2017)

[15] Shmoish, M., German, A., Devir, N., Hecht, A., Butler, G., Niklasson, A., Albertsson-Wikland, K., et al.: Prediction of adult height by artificial intelligence (ai) through machine learning $(\mathrm{ml})$ from early height data. In: 57th Annual ESPE, vol. 89 (2018). European Society for Paediatric Endocrinology

[16] Amm, A., Am, A., Fa, B., Sg, A., Mm, B., Ac, C., Fd, C., Mh, E., Sl, D., Mjm, E.: Improved prediction equations for estimating height in adults from ethnically diverse backgrounds - sciencedirect. Clinical Nutrition 39(5), 1454-1463 (2020)

[17] Park, S.-h., Cho, Y.-b.: Convolution neural network based tw3 maximum height prediction system. Journal of the Korea Institute of Information 
and Communication Engineering 22(10), 1314-1319 (2018)

[18] Fan, D., Yang, J., Zhang, J., Lv, Z., Huang, H., Qi, J., Yang, P.: Effectively measuring respiratory flow with portable pressure data using back propagation neural network. IEEE journal of translational engineering in health and medicine $6,1-12$ (2018)

[19] Guo, H., Zeng, W., Shi, Y., Deng, J., Zhao, L.: Kernel granger causality based on back propagation neural network fuzzy inference system on fmri data. IEEE Transactions on Neural Systems and Rehabilitation Engineering 28(5), 1049-1058 (2020)

[20] Mirjalili, S.: The ant lion optimizer. Advances in engineering software 83, 80-98 (2015)

[21] Dinkar, S.K., Deep, K.: Opposition based laplacian ant lion optimizer. Journal of computational science 23, 71-90 (2017) 